\title{
Evaluation of Musculoskeletal Re-Injury Occurrence in Previously Concussed National Football League Athletes
}

This article was published in the following Dove Press journal: Open Access Journal of Sports Medicine

\author{
Emily M Wittrup' \\ Lucas A Fox' \\ Katherine $M$ Breedlove iD $^{2,3}$ \\ Allyssa K Memmini (D) \\ Steven P Broglio (D) \\ 'Concussion Center, University of \\ Michigan, Ann Arbor, MI, USA; ${ }^{2}$ Center \\ for Clinical Spectroscopy, Brigham and \\ Women's Hospital, Boston, MA, USA; \\ ${ }^{3}$ Department of Radiology, Harvard \\ Medical School, Boston, MA, USA
}

\begin{abstract}
Introduction: Increased risk of musculoskeletal (MSK) injury post-concussion has been reported in collegiate athletes, yet it is unknown if professional football athletes are at the same risk of secondary injury. The objective of this study was to determine if the risk of MSK injury in National Football League (NFL) athletes increases after concussion.

Methods: NFL injury reports from 2013 to 2017 were collected from public websites. Concussed athletes ( $\mathrm{n}=91$ ) were equally matched to a non-injured control and an athlete with an incident of musculoskeletal (MSK) injury.

Results: Following their return to sport, concussed athletes were 2.35 times more likely to have a subsequent MSK injury relative to non-injured controls (95\% CI: 2.35 [1.25, 4.44], $P=0.01)$, but were no more likely than athletes with an incident MSK injury $(P=0.55)$. Likewise, athletes with an incident MSK injury were no more likely to have a subsequent MSK injury than controls $(P=0.08)$.

Discussion: Increased odds of MSK injury in the 12-week period following a concussion in professional football athletes warrants future research on the acute effects of concussion and the relationship to MSK injury risk.
\end{abstract}

Keywords: mild traumatic brain injury, musculoskeletal injury, return to play, NFL, professional American football

\section{Introduction}

Approximately 1.6 to 3.8 million sport- and recreation-related traumatic brain injuries occur every year. ${ }^{1}$ The acute effects of concussion are well defined, with notable changes in postural stability due to impaired integration of the visual, vestibular, and somatosensory systems. ${ }^{2}$ The sensory integration deficit can present clinically as impaired execution of motor tasks, ${ }^{3}$ such as gait, balance and delayed reaction time. ${ }^{4}$ Historically, it was believed that motor impairments associated with acute concussion returned to pre-morbid levels shortly after injury. ${ }^{5}$ More recent investigations have suggested secondary sports-related concussion (SRC) effects, including increased musculoskeletal (MSK) injury risk following acute SRC resolution. ${ }^{6-8}$

The biological underpinnings of increased injury rates post-SRC are not clear, but collegiate athletes, across numerous sports, had an increased odds of MSK injury in the 90-day period after return to play from a SRC compared to matched controls. $^{6,7}$ Recently, Harada et al investigated the relationship between SRC and lower extremity injury risk over a 16-year timeframe in Division I collegiate athletes. Researchers concluded those who sustained multiple SRCs were three
Correspondence: Emily M Wittrup Michigan, 40I Washtenaw Avenue, Ann Arbor, MI 48109, USA

Tel + $810-986-5105$

Fax +I 734-936-1925

Email ewittrup@umich.edu 
times more likely to sustain lower extremity injuries compared to those who sustained a single concussion and healthy controls. ${ }^{8}$

Despite previous work suggesting an increased risk for MSK injury following SRC in collegiate athlete populations, ${ }^{6-8}$ how these effects may influence professional football athletes is less clear. Since National Football League (NFL) athletes participate in a longer competitive season compared to their collegiate counterparts (17 weeks versus 10-13 weeks, respectively) it follows that the number of athlete exposures also increases for professional athletes. There is limited literature regarding injury risk in terms of athlete exposure in professional American football athletes. ${ }^{9,10}$ Direct comparison between the professional and collegiate population is difficult due to varying definitions of athlete exposure between studies. However, an increase in athlete-exposures in NFL athletes intuitively leads to a greater risk of MSK injuries and SRCs, the relationship of which has yet to be thoroughly investigated. In 2015, Pietrosimone et al conducted a survey of retired NFL athletes, and found those reporting three or more SRCs during their career were between $136 \%$ and $248 \%$ more likely to report a lower extremity MSK injury than athletes reporting zero SRCs. ${ }^{11}$ Given this result, it was hypothesized there would be an increased risk of MSK injury after returning to sport following a SRC. Therefore, the purpose of this study is to determine the risk of MSK injury in recently concussed NFL athletes after return to play, compared to those who sustained an acute MSK injury at the same point in the season and healthy controls.

\section{Methods}

\section{Participants}

A retrospective cohort study was conducted to evaluate the occurrence of MSK injuries in concussed and non-concussed athletes in the NFL. Injury reports from all 32 NFL teams were obtained from an online public source from the 2013-2017 regular and post-season games (The Football Database, www.footballdb.com; Fox Sports NFL Injuries, www.foxsports.com/nfl/injuries; accessed October 2018). This study was conducted as secondary research meeting federal exemption requirements from the University of Michigan's Medical Institutional Review Board. Preseason injuries were excluded from our dataset as they were not required to be reported by the NFL's 2015-2017 "Personnel (Injury) Report Policy". The resulting database included
10,862 injuries from 6146 individual athlete seasons. Exclusion criteria of 1073 athlete seasons included: nonathletic-related injuries, general medical or illness, head or neck injury not labeled as an SRC, undisclosed injury, or an SRC concurrent with an MSK injury. Athletes were grouped into seven general position categories: defensive back, quarterback, linebacker, wide receiver, tight end, running back, and lineman.

Concussions were identified as defined by the injury report $(n=683)$ and included for analyses only if return to play (RTP) occurred between the third and eighth week of the regular season $(n=186)$. This approach was taken to avoid analysis of SRCs that occurred in the pre-season, as well as to ensure at least nine remaining weeks of the regular season following RTP to calculate injury occurrences.

Two certified athletic trainers manually classified all MSK injuries $(\mathrm{N}=8836)$ from the injury reports of each of the 32 teams, as either repetitive $(n=2100)$ or acute $(n=6736)$ based on location and mode of onset. ${ }^{12}$ Both upper and lower extremity injuries were included since SRC could lead to motor cortex dysfunction which disposes an athlete to a wide range of injuries, not limited to lower extremity. ${ }^{13}$ Repetitive injuries were defined by a mechanism indicative of long-term repeated injury and were thus excluded from matching due to the inability to distinguish if injuries occurred from SRC or a pre-existing susceptibility. ${ }^{14}$ This study is a secondary analysis of publicly available data and therefore meets the federal exemption requirements from the Institutional Review Board at the University of Michigan.

\section{Participant Matching}

Each SRC athlete was matched with two control athletes as illustrated in Figure 1. They were matched on team, season, and position to an athlete who sustained an MSK injury based on time lost (i.e. RTP within two weeks of the concussed athlete's RTP) and second to a healthy control who did not report an injury in the three weeks prior to the SRC athlete's injury. If MSK and control athletes could not be found, the SRC athlete was excluded. This resulted in a final sample of 273 participants split equally among the three groups: $\mathrm{SRC}(\mathrm{n}=91)$, MSK control ( $n=91)$, and healthy control $(n=91)$ athletes. Participant matching was conducted using Python (version 3.7.3; Python Software Foundation, Wilmington, DE) programming language. 


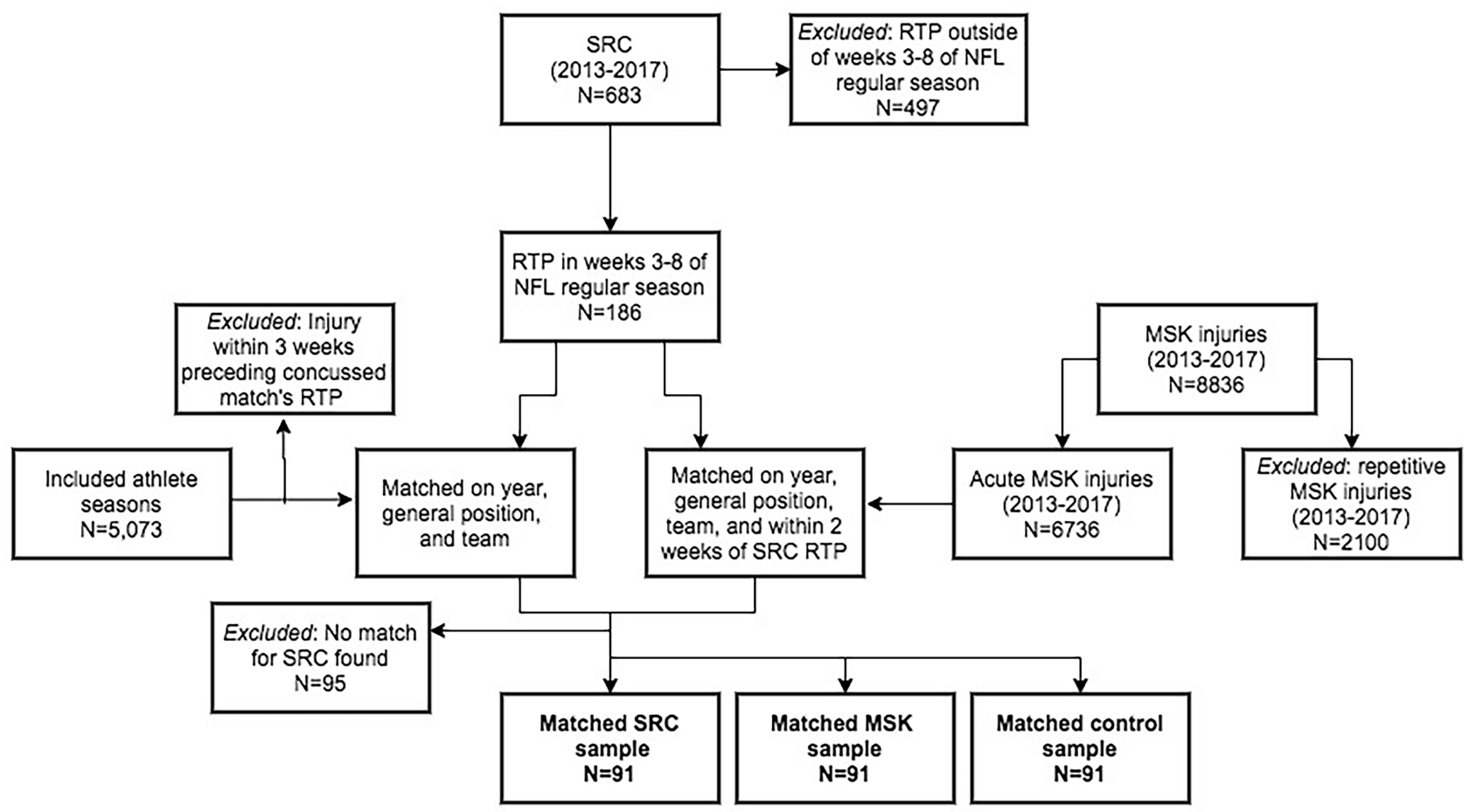

Figure I Illustration of matching of SRC to MSK and control athletes, followed by reasons for injury exclusions.

Notes: Of the 683 athletes who sustained an SRC, only 186 returned to play during the third through eighth week of the regular NFL season, and 9 I could be matched with appropriate MSK and healthy controls.

Abbreviations: SRC, sports-related concussion; MSK, musculoskeletal; NFL, National Football League; RTP, return to play.

\section{Reinjury Occurrence}

MSK occurrence following the incident SRC or MSK injury was calculated by counting all subsequent MSK injuries (including repetitive, acute and cervical pathologies) up to 12 weeks post-RTP for each athlete, including at least 9 or 8 weeks of the regular season for SRC and MSK athletes, respectively. If the 12 weeks post-RTP window extended into the post-season, all subsequent MSK injuries within the window were counted until either the team was eliminated from the playoffs or the Conference Championships were completed. MSK injury counts for the matched healthy controls began on the week of return for the SRC athlete and was equivalent in duration. The post-injury follow-up is consistent with previous studies regarding injury surveillance. ${ }^{6,7}$

\section{Statistics}

Demographics for players with incident SRC, MSK and controls were compared using a one-way multivariate analysis of variance (ANOVA) to identify significant differences in confounding factors between groups. The Wilcoxon rank-sum test was used to calculate the pairwise difference in the number of MSK injuries between groups in the 12 weeks following RTP from their initial injury. This analysis was necessary since homogeneity of variance was violated and data were not normally distributed. To reduce the impact of players with multiple injuries, Fisher's exact test was used to calculate pairwise odds ratios for the existence of any MSK injury in the 12 weeks following RTP. Standardized differences for comparisons were analyzed using Cohen's $d$ effect size $(d)$ using the following thresholds: $\leq 0.2$ small, $0.2-0.5$ medium, $\geq 0.8$ large. ${ }^{15}$ All analyses were conducted using MATLAB (version 9.2.0; MathWorks, Inc., Natick, MA) statistical software and the significance threshold was set at 0.05 .

\section{Results}

Demographic information regarding SRC, MSK, and control matched samples are presented in Table 1. The ANOVA test showed no significant differences in the age, height, and mass between groups $(P>0.05)$. Table 2 presents the distribution of the matched sample by position, week of RTP, weeks missed and location of original injury. The majority of the matched sample 
Table I Player Demographics by Injury Type

\begin{tabular}{|c|c|c|c|c|c|}
\hline & Total $(N=273)$ & SRC (N=9l) & MSK (N=9l) & Control $(\mathrm{N}=91)$ & $P$-value \\
\hline Age in years, mean (SD) & $25.99(2.90)$ & $25.54(2.55)$ & $26.07(3.15)$ & $26.36(2.96)$ & 0.15 \\
\hline Age range, yr & $21-36$ & $21-33$ & $21-36$ & $21-34$ & \\
\hline Height in meters, mean (SD) & $\mathrm{I} .87(0.07)$ & $\mathrm{I} .88(0.07)$ & $\mathrm{I} .87(0.06)$ & $1.87(0.06)$ & 0.43 \\
\hline Height range, $\mathrm{m}$ & $1.73-2.03$ & $1.75-2.03$ & $1.75-2.01$ & $1.73-2.01$ & \\
\hline Mass in kilograms, mean (SD) & 108.37 (20.77) & $108.24(20.82)$ & $108.69(21.66)$ & $108.17(20.02)$ & 0.98 \\
\hline Mass range, $\mathrm{kg}$ & $77.56-154.22$ & $77.56-149.23$ & $79.38-154.22$ & $78.47-|52.4|$ & \\
\hline
\end{tabular}

Notes: One-way ANOVA comparing means of SRC, MSK, and control demographics proved to be non-significant $(P>0.05)$.

Abbreviations: SRC, sports- related concussion; MSK, musculoskeletal injury; yr, year; m, meters; kg, kilograms; SD, standard deviation.

$(80.3 \%)$ were defensive-backs, linemen, or linebackers. Running backs, tight ends, and wide receivers accounted for the remaining sample (19.7\%). A significant difference $(\mathrm{t}=2.35$; mean difference $=0.42$ weeks, $95 \%$ confidence interval [CI] 0.06 to $0.77 ; d=2.2, P=0.02$ ) was noted between the average number of weeks missed for a concussive injury (1.74 weeks) relative to the time missed for an MSK injury (2.15 weeks). The locations of MSK injuries for the MSK injury matches are detailed in Table 2.

In the 12 weeks following RTP, a significantly greater total number of subsequent MSK injuries occurred among the SRC athletes $(\mathrm{U}=5037.50, d=$ $0.3, P<0.01)$ and MSK athletes $(\mathrm{U}=4734.50, d=0.2$, $P=0.04)$ relative to the healthy control athletes (Figure 2). However, there was no difference in number of subsequent MSK injuries between the MSK and SRC athletes $(\mathrm{U}=3798.50, d=0.1, P=0.27)$. The Fisher's exact test suggested SRC athletes were 2.35 times more likely to have a subsequent MSK than healthy controls (odds ratio [OR] 2.35, 95\% CI 1.25 to $4.44 ; P=0.01$ ), but not more likely than athletes with an initial MSK injury (OR $0.80,95 \%$ CI 0.44 to $1.44 ; P=0.55$ ). Those with an initial MSK were not at greater risk for subsequent MSK than controls (OR 1.87, 95\% CI 0.99 to 3.55; $P=0.08$ ) (Table 3 ).

\section{Discussion}

Based on our findings, athletes sustaining an MSK injury did not have an increased odds of sustaining a subsequent MSK injury in the 12-week period following RTP than controls matched by team, year, and position. However, considering multiple MSK injuries per athlete during this same period, athletes sustaining an
MSK injury had significantly more MSK injuries than their matched controls. This result validates prior literature that previous MSK injuries can be a risk factor for subsequent injury. ${ }^{16-19}$ Alternatively, athletes sustaining an SRC were 2.35 times more likely to sustain a MSK injury and sustained significantly more MSK injuries than their matched healthy controls in the 12 weeks following RTP.

Although there is previous research suggesting greater risk of MSK injury when an athlete returns to play after sustaining a SRC, $6,8,11$ the current study provides insight into the conditional occurrence of MSK injury acutely following SRC in a professional athlete cohort. These results are consistent with previous studies in collegiate populations. ${ }^{6-8}$ Brooks et al reported a 2.48-fold increased risk of acute lower extremity MSK injury among recently concussed collegiate athletes across seven different sports compared to controls. ${ }^{6}$ Further, Herman et al reported collegiate athletes, across numerous sports, were at a 3.79 times greater risk of lower extremity MSK injury in the 90-days following RTP from SRC. ${ }^{7}$ The results presented here reflect those of Pietrosimone et al in the same population, in such that athletes reporting one, two, or more concussions during their career were, respectively, 1.59, 2.29, 2.86 times more likely to report lower extremity MSK injuries relative to former NFL athletes who did not report a concussion in their career. ${ }^{11}$

The results reported herein support a growing body of literature demonstrating an increased risk of MSK injury after an SRC or MSK injury. Following an $\mathrm{SRC}$, it is the standard of care to use clinically objective measures in determining when an athlete is ready to return to sport. $^{20}$ Indeed, medical professionals base 
Table 2 Position Type and Injury Characteristics

\begin{tabular}{|c|c|c|c|c|}
\hline & Total $(\mathbf{N}=273)$ & $\operatorname{SRC}(n=91)$ & MSK (n=9I) & Control $(n=91)$ \\
\hline \multicolumn{5}{|c|}{ Position, number of players (\%) } \\
\hline Defensive Back & 90 (33.0\%) & $30(33.0 \%)$ & $30(33.0 \%)$ & $30(33.0 \%)$ \\
\hline Linebacker & 51 ( $18.7 \%)$ & $17(18.7 \%)$ & $17(18.7 \%)$ & $17(18.7 \%)$ \\
\hline Linemen & $78(28.6 \%)$ & $2628.6 \%)$ & $2628.6 \%)$ & $2628.6 \%)$ \\
\hline Running Back & $12(4.4 \%)$ & $4(4.4 \%)$ & $4(4.4 \%)$ & $4(4.4 \%)$ \\
\hline Tight End & $9(3.3 \%)$ & $3(3.3 \%)$ & $3(3.3 \%)$ & $3(3.3 \%)$ \\
\hline Wide Receiver & $33(12.1 \%)$ & II (I2.I\%) & II (I2.I\%) & II (12.1\%) \\
\hline Quarterback & $0(0.0 \%)$ & $0(0.0 \%)$ & $0(0.0 \%)$ & $0(0.0 \%)$ \\
\hline \multicolumn{5}{|c|}{ Week of RTPa , number of players (\%) } \\
\hline $2-4^{b}$ & $60(22.0 \%)$ & $32(35.2 \%)$ & $28(30.8 \%)$ & $0(0.0 \%)$ \\
\hline $5-6$ & $53(19.4 \%)$ & $25(27.5 \%)$ & $28(30.8 \%)$ & $0(0.0 \%)$ \\
\hline $7-9^{c}$ & $69(25.3 \%)$ & 34 (37.4\%) & 35 (38.5\%) & $0(0.0 \%)$ \\
\hline No original injury & 91 (33.3\%) & $0(0.0 \%)$ & $0(0.0 \%)$ & $91(100.0 \%)$ \\
\hline \multicolumn{5}{|c|}{ Weeks missed $^{d}$, number of players (\%) } \\
\hline 1 & 89 (32.6\%) & 45 (49.5\%) & 44 (48.4\%) & $0(0.0 \%)$ \\
\hline 2 & $52(19.0 \%)$ & $34(37.4 \%)$ & $18(19.8 \%)$ & $0(0.0 \%)$ \\
\hline$\geq 3$ & $4 \mathrm{l}(15.0 \%)$ & $12(13.2 \%)$ & $29(31.9 \%)$ & $0(0.0 \%)$ \\
\hline No original injury & 91 (33.3\%) & $0(0.0 \%)$ & $0(0.0 \%)$ & $91(100.0 \%)$ \\
\hline \multicolumn{5}{|c|}{ Location of original injury, number of players (\%) } \\
\hline Head (Concussion) & 91 (33.3\%) & $91(100.0 \%)$ & $0(0.0 \%)$ & $0(0.0 \%)$ \\
\hline Shoulder & $13(4.8 \%)$ & $0(0.0 \%)$ & $13(14.3 \%)$ & $0(0.0 \%)$ \\
\hline Arm & $8(2.9 \%)$ & $0(0.0 \%)$ & $8(8.8 \%)$ & $0(0.0 \%)$ \\
\hline Chest & $5(1.8 \%)$ & $0(0.0 \%)$ & $5(5.5 \%)$ & $0(0.0 \%)$ \\
\hline Hip/Pelvis & $2(0.7 \%)$ & $0(0.0 \%)$ & $2(2.2 \%)$ & $0(0.0 \%)$ \\
\hline Leg & 14 (5.I\%) & $0(0.0 \%)$ & $14(15.4 \%)$ & $0(0.0 \%)$ \\
\hline Knee & 23 (8.4\%) & $0(0.0 \%)$ & $23(25.3 \%)$ & $0(0.0 \%)$ \\
\hline Ankle & 17 (6.2\%) & $0(0.0 \%)$ & 17 (18.7\%) & $0(0.0 \%)$ \\
\hline Foot & 9 (3.3\%) & $0(0.0 \%)$ & 9 (9.9\%) & $0(0.0 \%)$ \\
\hline No original injury & 91 (33.3\%) & $0(0.0 \%)$ & $0(0.0 \%)$ & 91 (100.0\%) \\
\hline
\end{tabular}

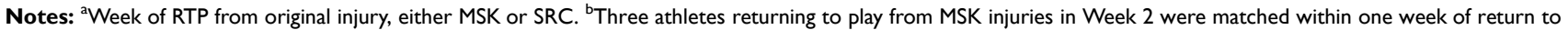
play to SRC athletes returning to play in Week $3 .{ }^{\mathrm{C}}$ Two athletes returning to play from MSK injuries in Week 9 were matched within one week of return to play to SRC athletes returning to play in Week $8 .{ }^{d}$ Weeks missed due to original injury, either MSK or SRC.

Abbreviations: SRC, sports-related concussion; MSK, musculoskeletal injury; RTP, return to play.

the post-concussion RTP progression on clinical data and the physical examination, but a growing body of work is suggests on-going, sub-clinical alterations that may extend beyond the clinical recovery time point. ${ }^{21}$ For example, Sosnoff et al (2011) concluded those with SRC history demonstrated altered postural dynamics compared to those controls, suggesting cerebral functionality directly associated with postural control may persist well beyond the acute timeline post-SRC. ${ }^{22}$ Furthermore, resting motor threshold, and intracortical inhibition and facilitation continue to be impaired long after clinical recovery from SRC. ${ }^{13}$ Findings included a positive, linear association between subsequent concussions and cortical silent periods, further indicating
SRC may lead to greater motor cortex dysfunction, which ultimately may result in increased risk of MSK injury. $^{23}$

The current study provides additional evidence for the association between concussion and MSK injuries by examining the period acutely following SRC for causality with subsequent MSK injuries. However, a major limitation of this study is the complex nature of injury reporting in the NFL which was used for this analysis. Undiagnosed injuries or those lasting less than a week may not be present on injury reports. Further, injuries occurring during the preseason or bye weeks were also not reported if the athlete recovered before the next regular season game. Injuries that 


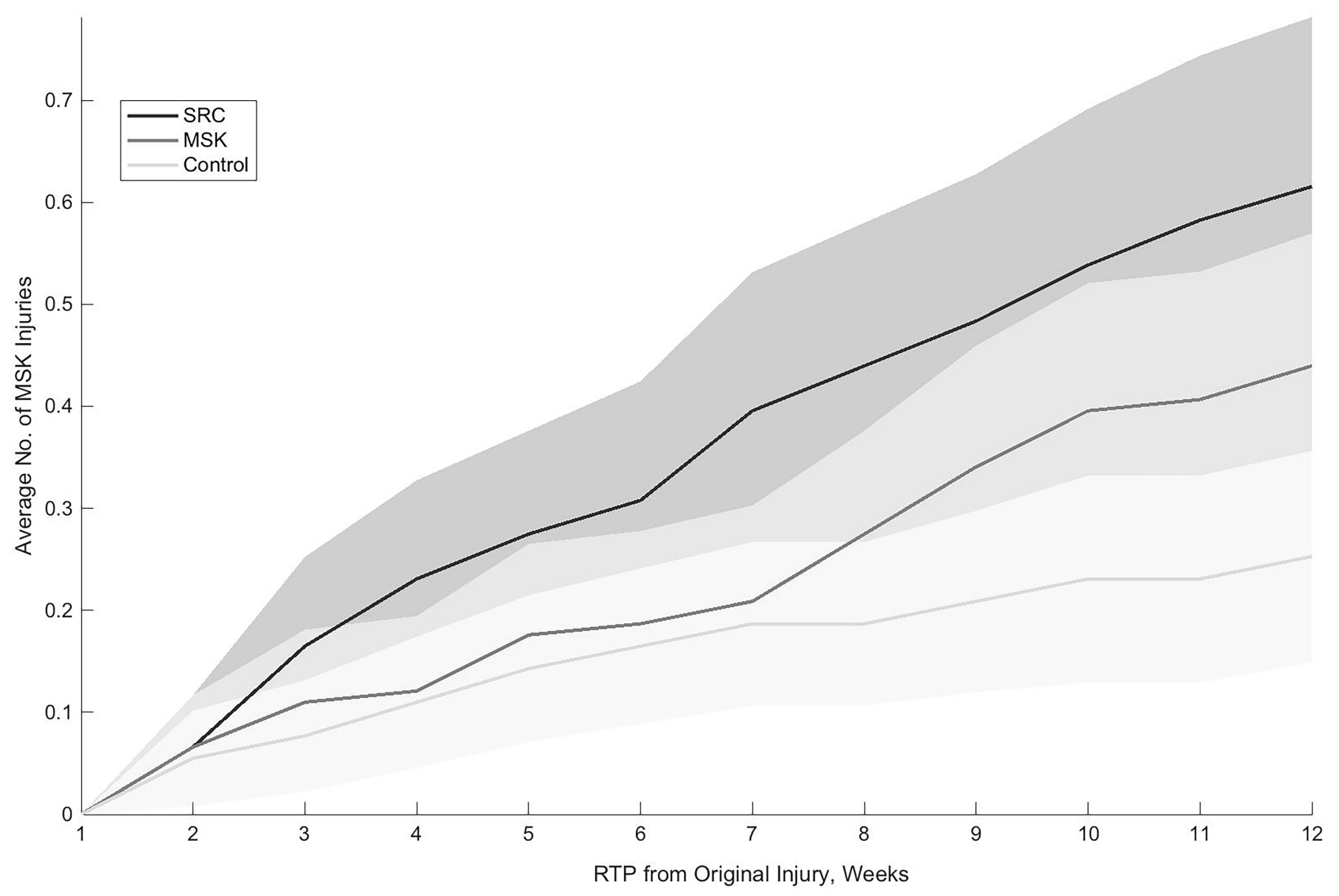

Figure 2 Average cumulative MSK injury occurrence for 12 weeks following RTP from original injury of SRC, MSK, or healthy controls.

Notes: SRC athletes 2.35 times more likely to sustain an MSK compared to controls. MSK athletes were 1.87 times more likely.

Abbreviations: MSK, musculoskeletal; RTP, return to play; SRC, sports-related concussion; No, number.

are reported lack detail, and comprehensive severity metrics outside of weeks missed. In addition, previous injuries were not considered which may influence the risk of MSK injuries. Additionally, it was assumed that injury deficits, whether they be due directly from injury or from sedentary behaviors following an MSK injury (such as a post-surgical athlete), may influence the risk of future injury. Due to this assumption, each successive SRC or MSK injury that followed an initial injury was considered to be conditional relative to the 12-week recovery period. Furthermore, each athlete was assumed to have equal playing time and therefore equal injury risk exposure, as some athletes are engaged in longer playing time than others which may ultimately increase their exposure to injury risk. Lastly, NFL bye-weeks were not taken into

Table 3 Reinjury Occurrences

\begin{tabular}{|l|l|l|l|l|}
\hline & Player (Co) & Injuries (Co) & Average Injuries per Player Mean (SD) & Fisher's Exact Test $^{\text {a }}$ OR(95\% CI) \\
\hline SRC & 39 & 59 & $0.65(0.87)$ & $2.35(1.25,4.44)^{*}$ \\
\hline MSK & 34 & 43 & $0.47(0.67)$ & $0.08(0.44,1.44)$ \\
\hline Control & 22 & 25 & $0.27(0.52)$ & - \\
\hline Total & 95 & 127 & $0.47(0.72)$ & - \\
\hline
\end{tabular}

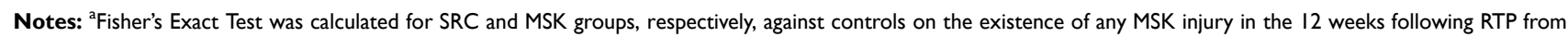
their original injury. *p $<0.05$.

Abbreviations: Co, count; SD, standard deviation; OR, odds ratio; $\mathrm{Cl}$, confidence interval; SRC, sports-related concussion; MSK, musculoskeletal injury. 
consideration for weeks missed calculations, which may have resulted in athletes with longer recovery timelines than what was reported on the original injury report. However, since athletes were matched on the same team, this should have minimal impact on the results.

\section{Conclusion}

Previous research has examined the impact of SRC on risk of re-injury in collegiate athletics, ${ }^{6-8}$ yet few have examined the potential for this risk in NFL athletes. ${ }^{11}$ Our findings suggest NFL athletes are more likely to sustain a MSK injury within the 12-week period following RTP from an SRC, relative to a non-injured control. These findings were present despite the implementation of a league wide concussion protocol beginning in 2013. As previously suggested, ${ }^{11}$ future work should evaluate this issue prospectively and, if replicated, develop interventions to mitigate risk. Additional research should also seek to define physiological recovery following SRCs and further develop objective guidelines for each stage of the RTP progression.

\section{Disclosure}

Dr Steven P Broglio reports grants from NCAA and funding from the Department of Defense of the Office of the Assistant Secretary of Defense for Health Affairs through the Psychological Health and Traumatic Brain Injury Program under Award no. W81XWH-14-2-0151, outside the submitted work; and Dr Broglio has current or past research funding from the National Institutes of Health; Centers for Disease Control and Prevention; Department of Defense - USA Medical Research Acquisition Activity, National Collegiate Athletic Association; National Athletic Trainers' Association Foundation; National Football League/Under Armour/GE; Simbex; and ElmindA. He has consulted for the NCAA (travel expenses only), US Soccer, US Cycling (unpaid), medicolegal litigation, and received speaker honorarium and travel reimbursements for talks given. He is on the University of Calgary SHRed Concussions external advisory board (unpaid) and is/was on the editorial boards (all unpaid) for Journal of Athletic Training (2015 to present), Concussion (2014 to present), Athletic Training \& Sports Health Care (2008 to present), British Journal of Sports Medicine (2008 to 2019). The author reports no other conflicts of interest in this work.

\section{References}

1. Langlois J, Rutland-Brown W, Wald M. The epidemiology and impact of traumatic brain injury. A brief overview. J Head Trauma Rehabil. 2006;21(5):375-378. doi:10.1097/00001199-200609000-00001

2. Guskiewicz KM, Ross SE, Marshall SW. Postural stability and neuropsychological deficits after concussion in collegiate athletes. $J$ Athl Train. 2001;36(3):263-273.

3. Miller NR, Yasen AL, Maynard LF, Chou LS, Howell DR, Christie AD. Acute and longitudinal changes in motor cortex function following mild traumatic brain injury. Brain Inj. 2014;28(10):1270-1276. doi:10.3109/ 02699052.2014.915987

4. Eckner JT, Kutcher JS, Broglio SP, Richardson JK. Effect of sport-related concussion on clinically measured simple reaction time. Br J Sports Med. 2014;48(2):112-118.

5. McCrea M, Guskiewicz KM, Marshall SW, et al. Acute effects and recovery time following concussion in collegiate football players: the NCAA Concussion Study. JAMA. 2003;290(19):2556-2563. doi:10.1001/jama.290.19.2556

6. Brooks MA, Peterson K, Biese K, Sanfilippo J, Heiderscheit BC, Bell DR. Concussion increases odds of sustaining a lower extremity musculoskeletal injury after return to play among collegiate athletes. Am J Sports Med. 2016;44(3):742-747.

7. Herman DC, Jones D, Harrison A, et al. Concussion may increase the risk of subsequent lower extremity musculoskeletal injury in collegiate athletes. Sports Med. 2017;47(5):1003-1010. doi:10.1007/ s40279-016-0607-9

8. Harada GK, Rugg CM, Arshi A, Vail J, Hame SL. Multiple concussions increase odds and rate of lower extremity injury in National Collegiate Athletic Association athletes after return to play. Am J Sports Med. 2019;47:363546519872502.

9. Lawrence DW, Hutchison MG, Comper P. Descriptive epidemiology of musculoskeletal injuries and concussions in the national football league, 2012-2014. Orthop J Sports Med. 2015;3 (5):2325967115583653.

10. Baker HP, Young-Hoon Lee K, Dayton SR, Terry M, Tjong VK. Thursday night football's impact on all-cause injuries in NFL players during 2012-2017. Phys Sportsmed. 2019;47(3):350-352. doi:10.1080/00913847.2019.1587565

11. Pietrosimone B, Golightly Y, Mihalik JP, Guskiewicz KM. Concussion frequency associates with musculoskeletal injury in retired NFL players. Med Sci Sports Exerc. 2015;47(11):2366-2372. doi:10.1249/MSS.0000000000000684

12. International Olympic Committee I, Illness Epidemiology Consensus G, Bahr R, et al. International Olympic Committee consensus statement: methods for recording and reporting of epidemiological data on injury and illness in sports 2020 (including the STROBE extension for Sports Injury and Illness Surveillance (STROBE-SIIS)). Orthop $J$ Sports $\quad$ Med. 2020;8 (2):2325967120902908.

13. Buckley TA, Baugh CM, Meehan WP, Difabio MS. Concussion management plan compliance: a study of NCAA power 5 conference schools. Orthop J Sports Med. 2017;5(4):232596711770260.

14. Knight K. More precise classification of orthopaedic injury types and treatment will improve patient care. J Athl Train. 2008;43(2):117-118. doi:10.4085/1062-6050-43.2.117

15. Cohen J. Statistical Power Analysis for the Behavioral Sciences. Academic press; 2013.

16. Chomiak J, Junge A, Peterson L, Dvorak J. Severe injuries in football players. Influencing factors. Am J Sports Med. 2000;28(5 Suppl): S58-S68. doi:10.1177/28.suppl_5.s-58

17. Hägglund $M$, Waldén $M$, Ekstrand J. Previous injury as a risk factor for injury in elite football: a prospective study over two consecutive seasons. Br J Sports Med. 2006;40(9):767-772. doi:10.1136/ bjsm.2006.026609 
18. Fulton J, Wright K, Kelly M, et al. Injury risk is altered by previous injury: a systematic review of the literature and presentation of causative neuromuscular factors. Int J Sports Phys Ther. 2014;9(5):583-595.

19. Hamilton GM, Meeuwisse WH, Emery CA, Steele RJ, Shrier I. Past injury as a risk factor: an illustrative example where appearances are deceiving. Am J Epidemiol. 2011;173(8):941-948. doi:10.1093/aje/ kwq461

20. Broglio SP, Cantu RC, Gioia GA, et al. National Athletic Trainers' Association position statement: management of sport concussion. J Athl Train. 2014;49(2):245-265. doi:10.4085/ 1062-6050-49.1.07
21. Churchill N, Hutchison M, Richards D, Leung G, Graham S, Schweizer TA. Brain structure and function associated with a history of sport concussion: a multi-modal magnetic resonance imaging study. J Neurotrauma. 2017;34(4):765-771. doi:10.1089/neu.2016.4531

22. Sosnoff JJ, Broglio S, Shin S, Ferrara M. Previous mild traumatic brain injury and postural-control dynamics. J Athl Train. 2011;46 (1):85-91. doi:10.4085/1062-6050-46.1.85

23. De Beaumont L, Lassonde M, Leclerc S, Theoret H. Long-term and cumulative effects of sports concussion on motor cortex inhibition. Neurosurgery. 2007;61(2):329. doi:10.1227/01.NEU.0000280000. 03578.B6

\section{Publish your work in this journal}

Open Access Journal of Sports Medicine is an international, peerreviewed, open access journal publishing original research, reports, reviews and commentaries on all areas of sports medicine. The manuscript management system is completely online and includes a very quick and fair peer-review system. Visit http://www.dovepress. com/testimonials.php to read real quotes from published authors. 